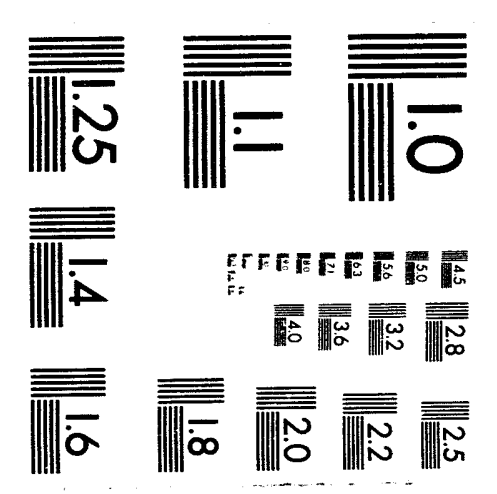



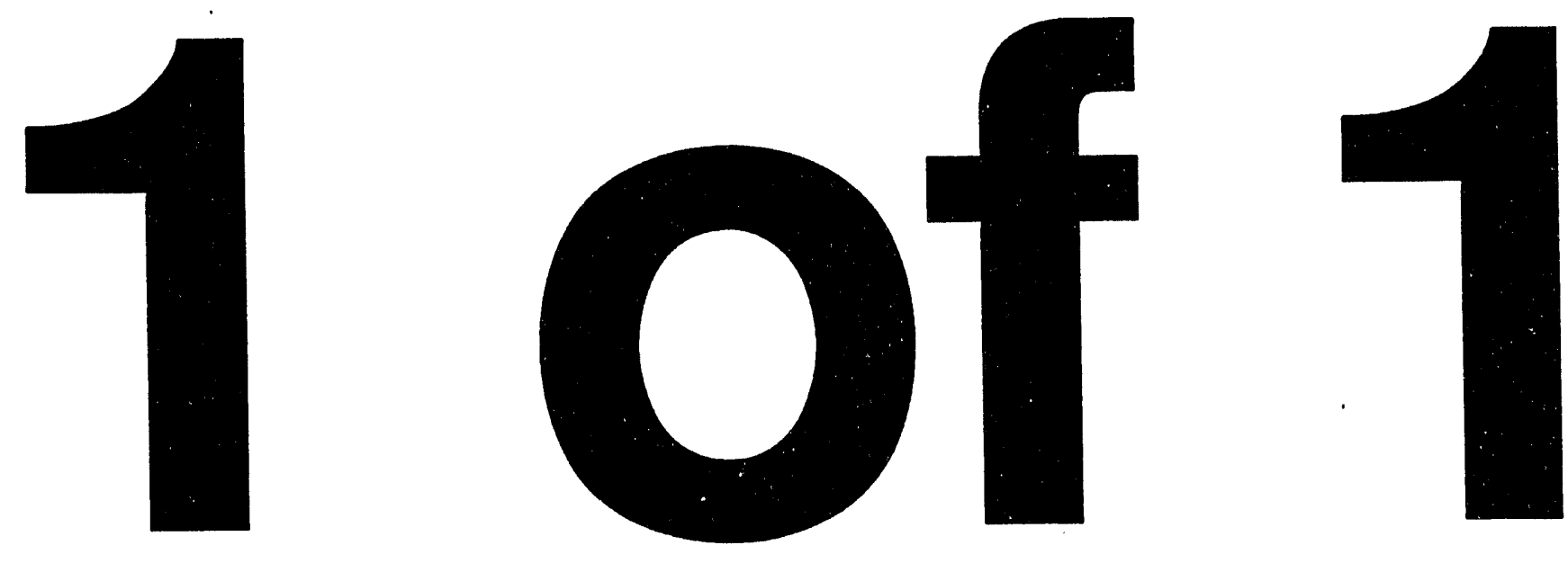


\section{Observation of the One- to Six-Neutron Transfer Resctions \\ at Sub-barrier Energies}

C. L. Jiang, K. E. Rehm, J. Gehring, B. Glagola,

W. Kutschera, M. Rhein and A. H. Wuosmaa

Physics Division, Argonne National Laboratory, Argonne IL 60439

Abstract

An unambiguous determination of the cross sections for the one- to six neutron transfer reactions has been made in the system ${ }^{58} \mathrm{Ni}+{ }^{100} \mathrm{Mo}$. The cross sections for multi-neutron transfer processes show an exponential falloff in agreement with recent theoretical calculations. Upper limits for the absolute yields to the ground states have been extracted which are smaller by a factor of ten as compared to theoretical predictions.

\section{DISCLAIMER}

This report was prepared as an account of work sponsored by an agency of the United States Government. Neither the United States Government nor any agency thereof, nor any of their employees, makes any warranty, express or implied, or assumes any legal liability or responsibility for the accuracy, completeness, or usefulness of any information, apparatus, product, or process disclosed, or represents that its use would not infringe privately owned rights. Reference herein to any specific commercial product, process, or service by trade name, 1.rademark, manufacturer, or otherwise does not necessarily constitute or imply its endorsement, recommendation, or favoring by the United States Government or any agency thereof. The views and opinions of authors expressed herein do not necessarily state or reflect those of the United States Government or any agency thereof.

\section{MASTER}

DISMHEUTION OF THIS DOCUMENT IS INMLAAITE
The submitted manuscript has been authored by a contractor of the U.S. Government under contract No. W-31-109ENG-38. Accordingly, the U.S. Government retains : Accordingly, the U.S. Government retains nonexclusive, rovalty-free license to publish or reproduce the published form of this
contribution, or allow others to do $\infty$, for U. S. Government purposes. 
During the last 20 years there has been continuous interest, both theoretically and experimentally in the study of multi-neutron transfer reactions. It has been suggested many years ago [1-3] that when two heavy nuclei are in contact during a grazing collision the transfer of several correlated neutron-pairs could occur. Most calculations performed so far have concentrated on superfluid Sn nuclei, and several experiments with Sn-isotopes have been performed. Despite of considerable experimental effort, however, only cross sections up to the four-neutron transfer reaction have been uniquely identified, although for multi-proton transfer reactions, yields for the transfer of up to six protons have been measured in the system ${ }^{144} \mathrm{Sm}+208 \mathrm{~Pb}$ [4].

The main difficulties in the study of multi-neutron transfer reactions are the small cross sections encountered at incident energies close to the barrier, and various experimental uncertainties which can complicate the anaiysis of these reactions. In a recent experiment in the system ${ }^{124} \mathrm{Sn}+{ }^{76} \mathrm{Ge}$ using $\boldsymbol{\gamma}-\boldsymbol{\gamma}$ coincidence techniques [5] $\boldsymbol{\gamma}$-ray transitions in ${ }^{118} \mathrm{Sn}$ have been observed. In this case it is not clear, however, to what extent these transitions originate from a four-neutron transfer to high-lying states in ${ }^{120} \mathrm{Sn}$ followed by the subsequent evaporation of two neutrons. The same uncertainty exists in an experiment using radiochemical techniques [6], where the detection of an ${ }^{110} \mathrm{Sn}$ activity does not necessarily prove the observation of the six-neutron transfer reaction ${ }^{116} \mathrm{Sn}\left({ }^{58} \mathrm{Ni},{ }^{64} \mathrm{Ni}\right){ }^{110} \mathrm{Sn}$, since ${ }^{110} \mathrm{Sn}$ can also originate from a fourneutron transfer reaction ${ }^{116} \mathrm{Sn}\left({ }^{58} \mathrm{Ni},{ }^{62} \mathrm{Ni}\right)$ followed by the subsequent evaporation of two neutrons.

An earlier investigation using the particle detection technique studied the production of heavy ( $\mathrm{A}>59$ ) $\mathrm{Ni}$-isotopes in the system ${ }^{124} \mathrm{Sn}+58_{\mathrm{Ni}}[7]$. In this experiment, however, the mass resolution of $A / \Delta A=70$ was not good enough to 
uniquely separate the isotopes of interest. The only experiment where a clear signature for four-neutron transfer has been observed was performed in the system ${ }^{112} \mathrm{Sn}+{ }^{120} \mathrm{Sn}[8]$. The main difficulty in this experiment was the possible interference from stable sn recoil particles which required the use of highly enriched target material. For instance, the detection of ${ }^{116} \mathrm{Sn}$ ions could be caused by ${ }^{116} \mathrm{Sn}$ recoil particles from a non-negligible fraction of ${ }^{116} \mathrm{Sn}$ in the enriched ${ }^{112} \mathrm{Sn}$ target material. This effect has already been demonstrated to influence one-neutron transfer reactions, as shown e.g. for the system $58_{\mathrm{Ni}}+{ }^{162} \mathrm{Dy}[9]$. In this paper we report the firsi measurement covering the full sequence from one- to the six-neutron transfer reactions at sub-barrier energies in the system ${ }^{58} \mathrm{Ni}+{ }^{100}$ Mo including angular distributions and Q-value spectra.

In order to eliminate the experimental difficulties mentioned above we have selected the system $58 \mathrm{Ni}+{ }^{100} \mathrm{Mo}$. To obtain single-mass and $\mathrm{Z}$ resolution at low incident energies where enhancements in the transfer cross sections due to pairing effects are expected, inverse reaction kinematics were used, i.e. a ${ }^{58_{\mathrm{Ni}}}$ target was bombarded with 100 Mo beams. With inverse kinematics, the Ni-like reaction products from central collisions are emitted at forward angles with sufficient energies which permit unique identification of their mass and atomic number 2. ${ }^{58} \mathrm{Ni}$ is available in highly enriched form and, in contrast to $\mathrm{Sn}$, shows almost no deterioration during heavy ion bombardment. From the Q-value systematics [10] one expects the transfer of neutrons from $100 \mathrm{Mo}$ to $58 \mathrm{Ni}$ to dominate the reaction yield. The detection of $59-64 \mathrm{Ni}$ ions at forward angles is thus a signature for one-to six-neutron transfer reactions. Since we are searching for isotopes which are heavier than the target, the problem with transfer followed by neutron evaporation is eliminated. 
The experiment was performed at the Argonne superconducting linear accelerator ATLAS with $100 \mathrm{Mo}$ beams of $380.4 \mathrm{MeV}$ obtained from the positive-ion injector. The average $\mathrm{cm}$-energy was $6.1 \mathrm{MeV}$ below the interaction barrier of 143.6 MeV, as calculated from the systematics of Vaz, Alexander and satchler [11]. The target consisted of a highly (>99.87) enriched $58_{\mathrm{Ni}}$ foil with a thickness of $334 \mu \mathrm{g} / \mathrm{cm}^{2}$. The isotopic abundances for $60,61,62,64 \mathrm{Ni}$ were 0.09 , $0.005,0.008$ and $0.02 \%$, respectively. The scattered particles were anslyzed according to their magnetic rigidity in a split-pole spectrograph, and detected in a hybrid focal-plane detector which measured the position, time-of-flight, energy and nuclear charge $z$ [12]. From these four parameters the mass, nuclear charge, atomic charge state of the detected particle and as well as the reaction $Q$-value can be uniquely determined. The mass and $z$-resolutions were $A / \Delta A=150$ and $z / \Delta z=70$, respectively. The energy resolution was linited to approximately $3 \mathrm{MeV}$ by the energy straggling in the target. Thus no transitions to individual levels could be resolved. Up to 7 and 5 charge states for Mo and $\mathrm{Ni}$, respectively, were detected simultaneously in the focal plane, representing more than $80 \%$ of the total yield at each angle. Angular distributions were measured in the range $\theta_{1 \mathrm{ab}}=10-50^{\circ}$. Using both Mo-like and Ni-like reaction products this covered the $\mathrm{c} \cdot \mathrm{m}$. angle range of $25^{\circ}-160^{\circ}$. Two monitor detectors mounted on either side of the beam were used for relative normalization. Absolute cross sections were obtained by normalizing the most forward elastic yields to the corresponding Rutherford cross sections.

Because of the low incident energy the transfer reactions are dominated by neutron transfer processes. At $E_{\mathrm{cm}}=137.5 \mathrm{MeV}$ the contributions to the total transfer yields from one- and two-proton transfer leading to $Z=27$ (Co) and $Z=$ 26 (Fe) channels are $16 z$ and 77 , respectively. Fig. 1 shows a mass spectrum for the $z=28$ (Ni), $q=23^{+}$charge state measured at $\theta_{1 a b}=10^{\circ}$ which, in addition 
to the $A=58$ channel and a weak $57 \mathrm{Ni}$ one-neutron stripping yield, reveals contributions from one- to six-neutron pickup processes. Using cross sections for elastic scattering at the respective scattering angle and the percentages for the target contaminants given above, the following contributions from recoil particles to the transfer yields can be expected: $0\left({ }^{59} \mathrm{Ni}\right), 0.67\left({ }^{60} \mathrm{Ni}\right)$, $\left.0.17\left(^{61} \mathrm{Ni}\right), 0.57\left({ }^{62} \mathrm{Ni}\right), 0\left({ }^{63} \mathrm{Ni}\right), 8 z^{64} \mathrm{Ni}\right)$. The contributions from the target contaminants can thus be neglected. More evidence that the ${ }^{64} \mathrm{Ni}$ events do not originate from ${ }^{64} \mathrm{Ni}$ contaminants in the target comes from the measured angular distribution. For recoil particles an increase in the yield with increasing laboratory angle is expected, which is not observed experimentally for the ${ }^{64} \mathrm{Ni}$ channel.

The resulting angular distributions for the ${ }^{58} \mathrm{Ni}$ channel and for the one to six-neutron transfer channels are shown in Fig. 2. The angular distribution for 'elastic scattering' (including inelastic excitations) shows a smooth fall-off from the Rutherford cross section at $\theta=90^{\circ}$ and reaches a value of 0.3 at $\theta=$ $180^{\circ}$. Oscillations as predicted e.g. in the calculations of Ref [2] for the system $\mathrm{Sn}+\mathrm{Sn}$ are not observed. The dashed line is the result of a coupled channels calculation with the code PTOLEMY [13] including the first excited $2^{+}$ states in ${ }^{58} \mathrm{Ni}$ and ${ }^{100} \mathrm{Mo}$. The parameters of the optical potential are given in the caption. The solid lines for the transfer reactions serve to guide the eyes. It should be noted that the maximum of the angular distribution for the transfer reactions moves to larger angles (i.e. smaller distances of closest approach) with increasing number of transferred neutrons.

The Q-value spectra measured at $\theta_{1 a b}=15^{\circ}$ (integrated over the two strongest charge states) are shown in Fig. 3. With increasing number of transferred neutrons the centroid of the distributions moves to higher 
excitation energies and their widths increase considerably. The average excitation energy is about $2.5 \mathrm{MeV}$ for the $\left({ }^{58} \mathrm{Ni},{ }^{59} \mathrm{Ni}\right)$ reaction and saturates at values of about $16 \mathrm{MeV}$ for the four- to six-neutron transfer reaction. As can be seen from Fig. 3 only very small yields for transitions in the ground-state region are observed.

The energy- and angle-integrated cross sections for the one- to six-neutron transfer reactions are shown in Fig. 4. One observes a continuous exponential decrease in the yields with a decrease in cross section by a factor of $3.87 \pm$ 0.17 for each transferred neutron. It should be noted that the energyintegrated cross sections for the $2 n, 4 n$ and $6 n$ transfers which correspond to the 1,2 and 3 -neutron pair transfers show no enhancement with respect to the in $3 n$ and $5 n$ yields.

Although the energy resolution does not allow to extract cross sections for transitions to individual states upper limits for the transition strengths to the respective ground-states for the two-, four- and six-neutron transfer reactions can be given. These yields can then be compared with theoretical predictions involving similar systems. These upper limits are shown as arrows in Fig. 4. They reach from $1.4 \mathrm{mb}$ for the two-neutron reaction to $1.5 \mu \mathrm{b}$ for the six-neutron transfer. No theoretical calculations for the system ${ }^{58} \mathrm{Ni}+$ ${ }^{100}$ Mo have been performed yet, but the cross sections for similar systems e.g. ${ }^{48} \mathrm{Ca}+{ }^{124} \mathrm{Sn}[14],{ }^{40} \mathrm{Ca}+{ }^{116} \mathrm{Sn}[15]$ and ${ }^{124} \mathrm{Sn}+{ }^{124} \mathrm{Sn}$ [14] al1 range from 10-60 mb for the two-neutron transfer to 50-600 $\mu \mathrm{b}$ for the six-neutron case. These values are about one order of magnitude larger than the upper limits obtained from our experiment. The reason for this discrepancy is not clear. The nuclei $\mathrm{Ni}$ and Mo should be describable within the pairing model as well as $\mathrm{Ca}$ and $\mathrm{Sn}$, but a detailed comparison with calculations for the $\mathrm{Ni}+$ Mo system still remains to be done. 
While the existing calculations overpredict the cross sections for the ground-state transitions by about a factor of 10 , they are in remarkable agreement with the total (energy-integrated) cross sections. The same holds for the falloff of the cross sections with increasing number of neutron transfers. For the system ${ }^{124} \mathrm{Sn}\left({ }^{48} \mathrm{Ca},{ }^{50} \mathrm{Ca}\right)$ the calculated cross section is about $12 \mathrm{mb}$ while the experimental value for the two-neutron transfer reaction $100 \mathrm{Mo}\left({ }^{58} \mathrm{Ni},{ }^{60} \mathrm{Ni}\right)$ is $28.2 \mathrm{mb}$. The predicted [14] falloff with increasing neutron number is 12.9 per transferred neutron pair(averaged over all possible neutron transfer reactions) while the experimental value is $15 \pm 0.7$. The theoretical value from Ref. 14 normalized to the cross section for the two-neutron transfer reaction $\left(58_{\mathrm{Ni}},{ }^{60} \mathrm{Ni}\right)$ is shown as a dashed line in Fig. 4 and is in good agreement with the experimental data.

In summary, angular distributions and Q-value spectra for the one- to sixneutron transfer reactions in the system $58 \mathrm{Ni}+100 \mathrm{Mo}$ have been measured for the first time. The angular distributions show a smooth angle dependence without any indications of oscillations as predicted by some theoretical calculations. For ground state transitions upper limits for the two, four and six- neutron transfer cross sections have been extracted which are about one order of magnitude smaller than calculated for similar systems within the pairing model.

This work was supported by the U.S. Department of Energy, Nuclear Physics Division, under contract W-31-109-ENG-38. 
[1] K. Dietrich. Ann. Phys. 66, 480(1971)

[2] R. Dietrich et a1., Phys, Lett. 35B, 349(1971)

[3] M. Kleber and H. Schmidt, 2. Phys. 245, 68(1971)

[4] J. Speer et al., Phys. Lett. 259B, 422(1991)

[5] R. Broda et al., Phys. Rev. Lett. 68, 1671(1992)

[6] W. Dunnweber et a1., Phys. Lett. 106B, $47(1981$ ).

[7] I. Chiodi et al., Lett. al Nuovo Cim. 33, 159(1982)

[8] W. von Dertzen et a1., 2. Phys A326, 463(1987)

[9] J. S. Lilley et al., Daresbury Ann. Rep. 1989/90, unpublished

[10] R. E. Rehm, Ann. Rev. Nucl. Part. Sci 41, 429 (1991)

[11] L. C. Vaz et al., Phys. Lett. 69C, 373(1981)

[12] R. E. Rehm and F. L. H. Wolfs, Nucl. Instr. and Meth. A273, 262(1988)

[13] M. H. Macfarlane and S. C. Pieper, Argonne National Laboratory Report No. ANL-76-11(Rev.1), 1978 (unpublished)

[14] J. H. Sorensen and A. Winther, NBI preprint 92-49, to be pub1. in Nucl. Phys. A

[15] S. M. Lenzi et al., Phys. Rev. C44, 2670(1991) 
Fig. 1 Mass spectrum measured for particles with charge state $q=23^{+}$at $\theta_{1 a b}$ $=10^{\circ}$ in the system ${ }^{58} \mathrm{Ni}+100 \mathrm{Mo}$.

Fig. 2 Angular distributions for elastic scattering (including inelastic excitations) and for the one- to six-neutron transfer reaction reactions. The dashed line for the elastic scattering is the result of a coupled-channels calculation with the potential V $=79 \mathrm{MeV}, \mathrm{r}_{0}=1.19$ $\mathrm{fm}, a=0.63 \mathrm{fm}, W=40 \mathrm{MeV}, r_{i}=1.21 \mathrm{fm}$ and $a_{i}=0.61 \mathrm{fm}$. The lines for the transfer reactions serve to guide the eye.

Fig. 3 Q-value spectra measured for one- to six-neutron transfers at $\theta_{1 a b}=$ $15^{\circ}$, summed over the two strongest charge states.

Fig. 4 Angle- and energy-integrated cross sections for the one- to six-neutron transfer reaction in the system $58_{\mathrm{Ni}}+{ }^{100} \mathrm{Mo}$ (solid points). The solid line is the result of a least-squares fit to the data. The dashed line is the prediction from Ref. 14 normalized to the cross section of the two-neutron transfer reaction. The arrows are the upper limits for the ground-state transitions extracted from the Q-value spectra. 


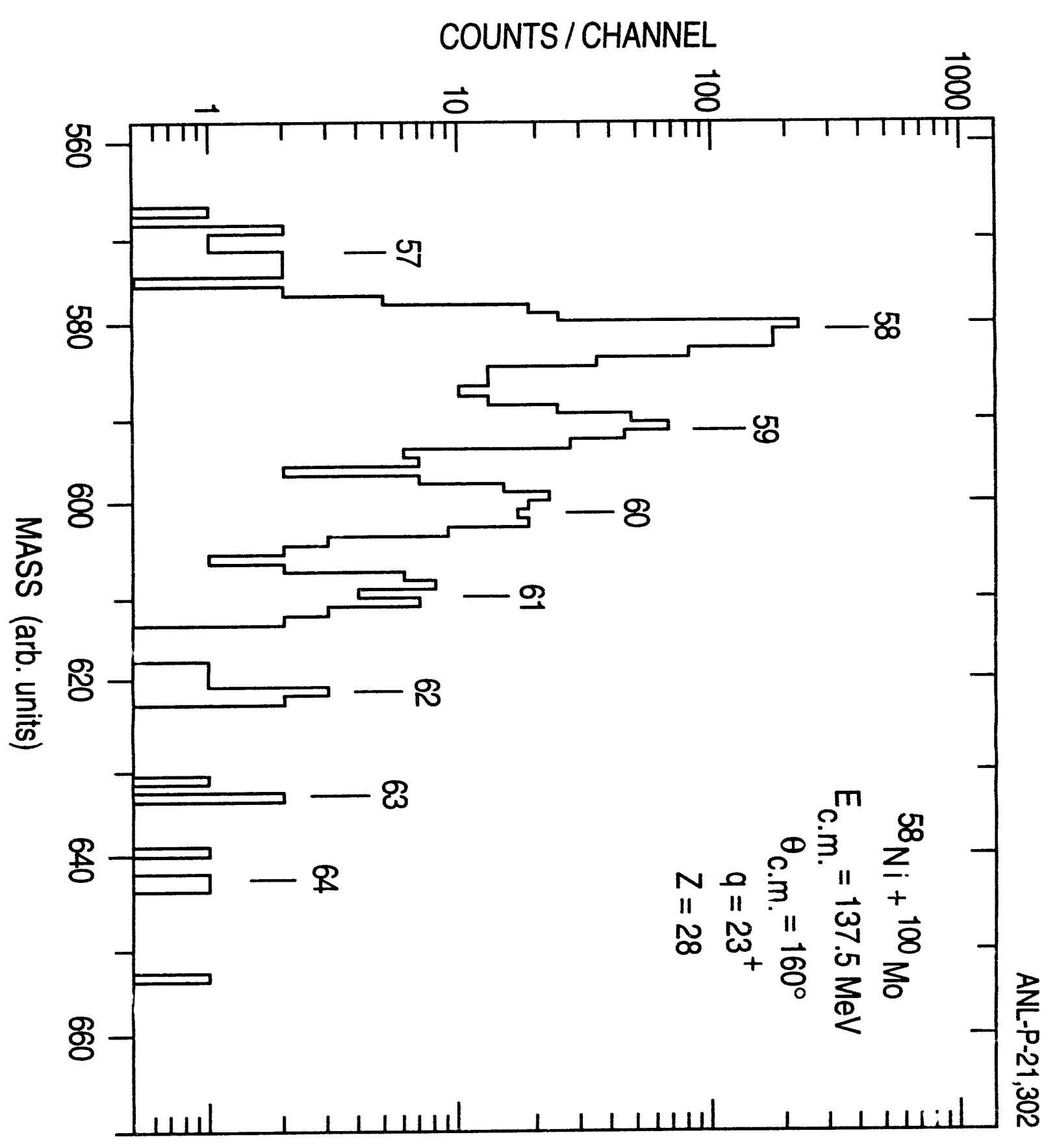




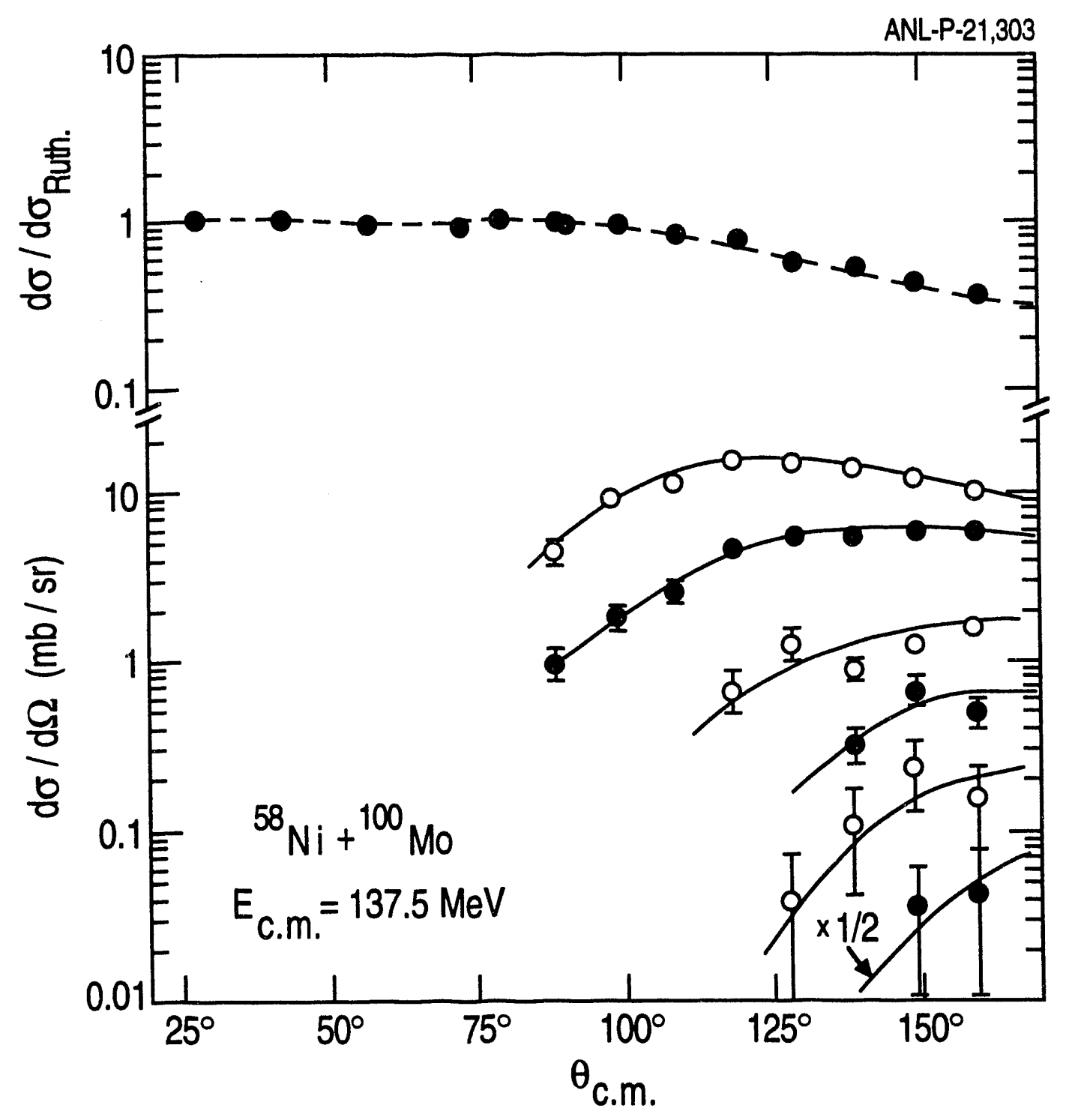

Fig. 2 


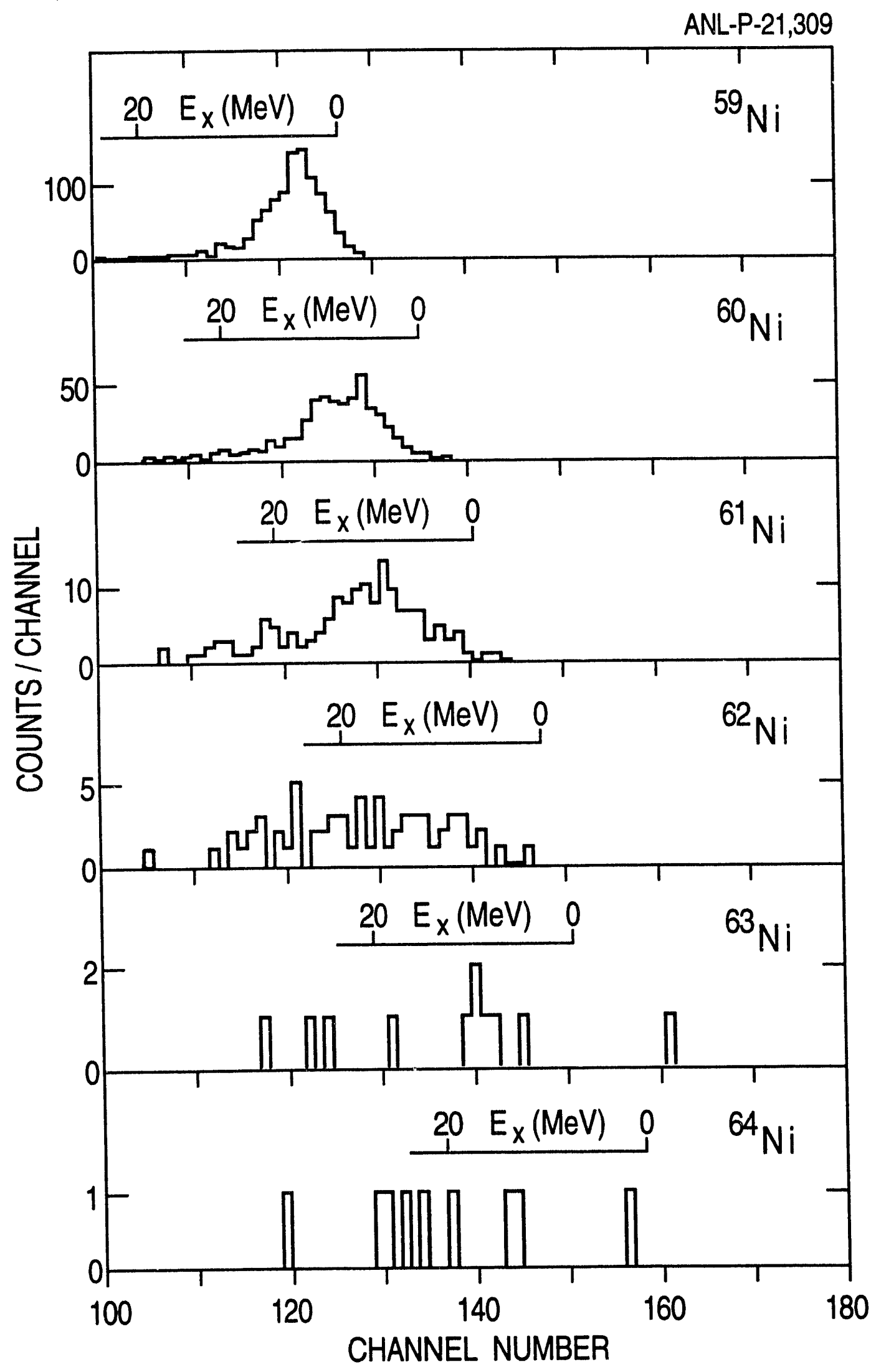

Fig. 3 


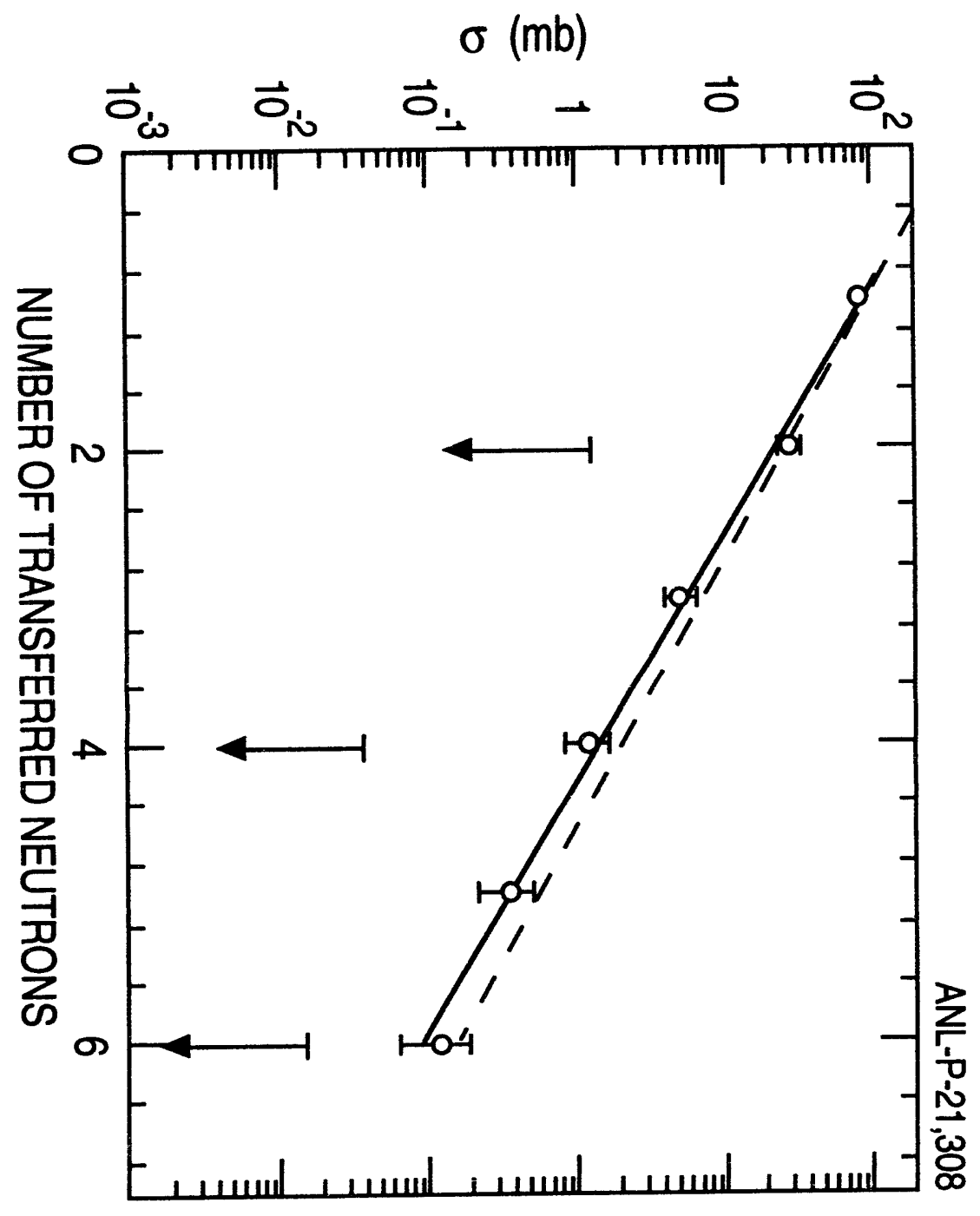




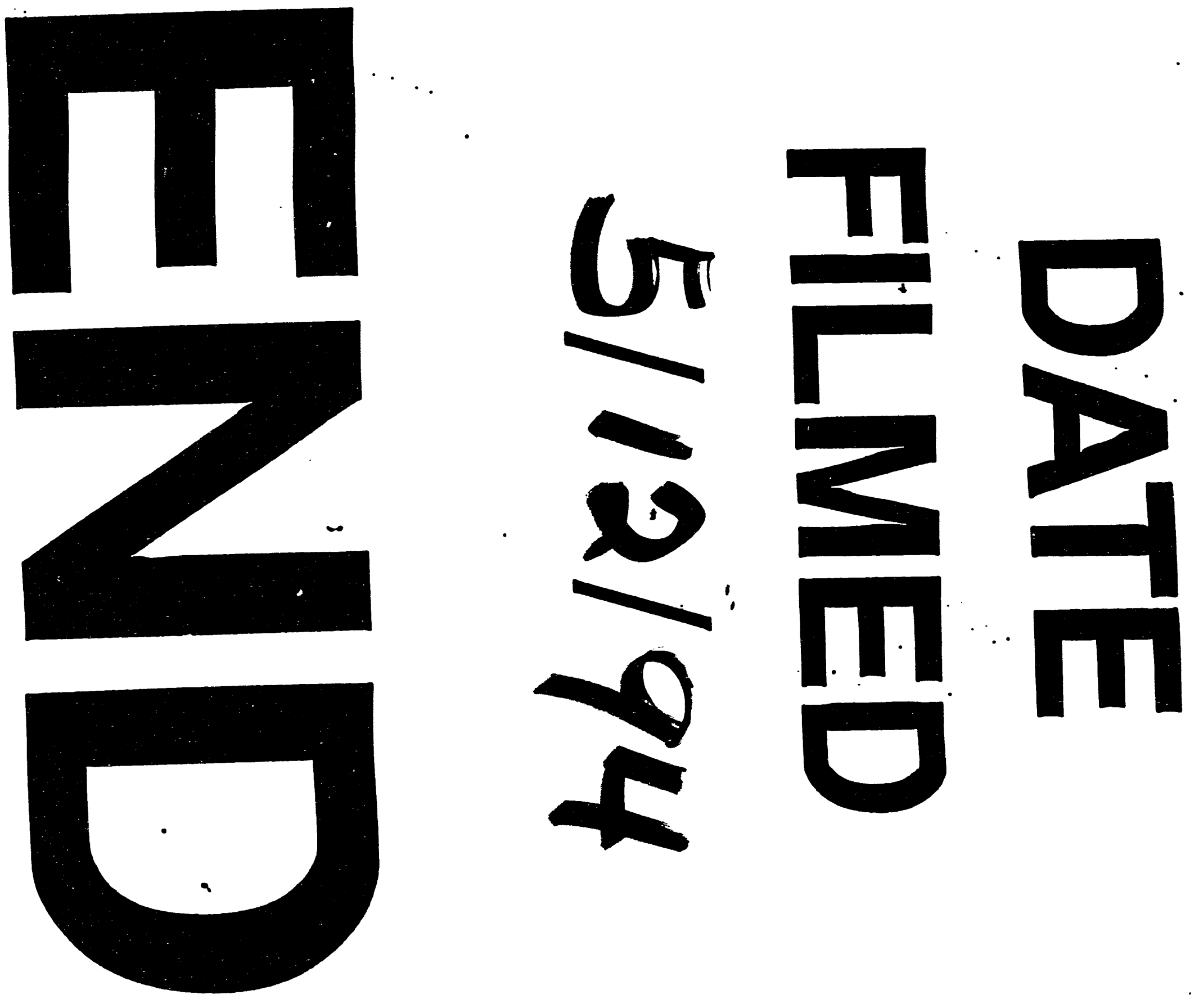


